Research Paper

\title{
Identification of PGC-related ncRNAs and their relationship with the clinicopathological features of Gastric Cancer
}

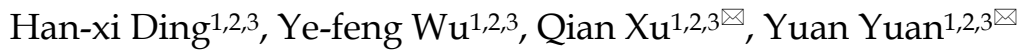 \\ 1. Tumor Etiology and Screening Department of Cancer Institute and General Surgery, the First Hospital of China Medical University, Shenyang 110001, \\ China. \\ 2. Key Laboratory of Cancer Etiology and Prevention in Liaoning Provincial Education Department, the First Hospital of China Medical University, Shenyang \\ 110001, China. \\ 3. Key Laboratory of GI Cancer Etiology and Prevention in Liaoning Province, the First Hospital of China Medical University, Shenyang 110001, China.
}

$\triangle$ Corresponding authors: Dr. YuanYuan, Tumor Etiology and Screening Department of Cancer Institute and General Surgery, the First Hospital of China Medical University, No. 155 NanjingBei Street, Heping District, Shenyang, Liaoning Province, P.R. China 110001. Telephone: +86-024-83282153; fax: +86-024-83282383; E-mail: yuanyuan@cmu.edu.cn. Dr. Qian Xu, Tumor Etiology and Screening Department of Cancer Institute and General Surgery, the First Hospital of China Medical University, No.155 NanjingBei Street, Heping District, Shenyang, Liaoning Province, P.R. China 110001. Telephone: +86-024-83282153; fax: +86-024-83282383; E-mail: qxu@cmu.edu.cn.

(1) The author(s). This is an open access article distributed under the terms of the Creative Commons Attribution License (https://creativecommons.org/licenses/by/4.0/). See http://ivyspring.com/terms for full terms and conditions.

Received: 2020.05.05; Accepted: 2021.04.22; Published: 2021.05.27

\begin{abstract}
Pepsinogen $C$ (PGC) is considered to be the final product of mature differentiated gastric mucosa. The expression level of PGC in gastric mucosa is clearly decreased upon the development of gastric cancer (GC). However, the mechanism behind PGC's down-regulation remains unclear and needs to be clarified. This study aimed to identify PGC-related ncRNAs with the potential to be PGC post-transcriptional regulators and to further explore the association between these ncRNAs and the clinicopathological parameters of GC. Bioinformatic software was used to predict miRNAs binding specifically to PGC and circRNAs binding specifically to these candidate miRNAs. Dual-luciferase reporter assay was performed to validate the completely complementary pairing of PGC and PGC-related ncRNAs. qRT-PCR was applied to determine the expression levels of PGC and PGC-related ncRNAs in GC tissue. hsa-let-7c was predicted to bind to the PGC gene, which was confirmed by dual-luciferase reporter assay. hsa_circ 0001483 and hsa_circ_0001324 were identified to bind to hsa-let-7c by bioinformatic analysis and dual-luciferase reporter assay. In addition, the hsa_circ_0001483/hsa_circ_0001324 -hsa-let-7c-PGC axis was confirmed in tissue by qRT-PCR. The expression level of hsa_circ_0001483 was correlated with peritumoral inflammatory cell infiltration and lymphatic metastasis. hsa_circ_0001483, hsa_circ 0001324, and let-7c were newly identified and validated as PGC-related ncRNAs and showed associations with the clinicopathological features of GC. The hsa_circ_0001483/hsa_circ_0001324-hsalet-7c-PGC axis in GC may account for the down-regulation of PGC in GC tissue.
\end{abstract}

Key words: CircRNA; MiRNA; Pepsinogen C; Gastric cancer; ceRNA network

\section{Introduction}

Pepsinogen C (PGC), one of the mature forms of pepsinogen (PG), belongs to the aspartic protease family and is activated under acidic conditions [1-3]. Under normal physiological conditions, PGC is mainly expressed in the whole stomach and is considered to be the final product of mature differentiated gastric mucosa [4]. Multiple studies have demonstrated that PGC could play important roles in maintaining the normal morphology and physiological function of gastric epithelial cells $[5,6]$. According to our previous research, the expression level of PGC changes significantly in pathological condition, for example, PGC expression in gastric mucosa was found to decrease considerably during the transformation from superficial gastritis (SG) to atrophic gastritis (AG), and was even absent in gastric cancer (GC) [2]. The low PGC level combined with the high expression of markers of a malignant phenotype 
such as MG7-Ag and MMP9 could be crucial molecular factors in the malignancy of gastric mucosa [6]. In addition, protein chip arrays and SELDI-TOF MS suggested that PGC was clearly reduced in GC compared with that in normal gastric mucosa, which was confirmed by 2D electrophoresis and immunohistochemistry [7]. Genome-wide association studies were conducted in the search for GC susceptibility loci and PGC was found to be a key gene in gastric epithelial differentiation. These findings indicated that PGC expression was negatively related to the occurrence and progression of GC [8]. However, the mechanism behind the downregulation of PGC remains unclear. Elucidation of how PGC gene expression is regulated could provide deeper insight into the pathogenesis of GC as well as aid in the identification of early diagnostic indicators and novel molecular targets for treating GC.

Non-coding RNAs (ncRNAs) play important roles in the post-transcriptional regulation of gene expression $[9,10]$. miRNAs are the most well-known ncRNAs, which are involved in the regulation of mRNA translation by binding to the $3^{\prime}$-untranslated region ( $3^{\prime}$-UTR), leading to the deregulation or suppression of mRNA transcripts [11, 12]. circRNAs are another kind of ncRNAs that are widely present in eukaryotic cells, showing differences in expression among tissues types $[13,14]$. Many circRNAs contain miRNA binding sites, which can competitively bind to miRNA response elements (MREs), thus sequestering miRNAs from their target genes and further increasing the expression levels of target genes $[13,15]$. Owing to their function as miRNA sponges, circRNAs are closely associated with miRNAs and the relationships in circRNA-miRNA-mRNA networks have attracted substantial interest.

As an effective molecular marker in GC progression, exploration of the regulation of PGC expression by upstream ncRNAs may provide new insights into the genesis and development of GC. To date, a few PGC-related ncRNAs have been investigated. Liu et al. found that the serum expression level of lncRNAs adjacent to PGC could distinguish gastric cancer, atrophic gastritis and superficial gastritis [16]. In addition, Lv et al. reported that 10 pairwise PGC-lncRNA single nucleotide polymorphisms (SNPs) were associated with gastric cancer risk, and might enhance the susceptibility to gastric cancer [17]. Several other studies focused on the miRNAs targeting PGC and their relationship with gastric cancer. Some SNP or SNP-SNP interactions of these miRNAs showed significant correlations with the risk or prognosis of gastric cancer [18, 19]. However, no evidence about the ncRNA network regulating PGC gene expression has yet been unearthed. In the present study, the ncRNAs involved in PGC regulation were predicted and verified, and the association between PGC-related ncRNAs and the clinicopathological characteristics of GC was further explored. This study showed help to clarify the regulatory mechanisms of PGC and provide clues for identifying ncRNA markers of GC diagnosis and prognosis.

\section{Materials and methods}

\section{Target miRNAs and circRNAs for PGC by bioinformatic analysis}

Two-stage bioinformatic analysis was performed to seek target miRNAs and circRNAs for PGC. First, the possible target miRNAs for PGC were predicted using eight software programs (i.e. MirTarBase, http:/ / mirtarbase.mbc.nctu.edu.tw/php/index.php; DIANA-microT, http://diana.imis.athenainnovation.gr/DianaTools/index.php? $\mathrm{r}=$ microtv4/in dex; MicroRNA.org, http://www.microrna.org/ microrna/getMirnaForm.do; miRDB, http://www. mirdb.org/; RNA22-HAS, https://cm.jefferson.edu/ rna22/; TargetMiner, https://www.isical.ac.in/ bioinfo_miu/targetminer20.htm; TargetScan-vert, http://www.targetscan.org/vert_71/ and PicTarvert, https://pictar.mdc-berlin.de/), and an miRNA was included when three or more software programs suggested that it might be a target ncRNA of the PGC gene [20-27]. In the prediction of possible target circRNAs for miRNAs, miRanda prediction algorithms (http://www.microrna.org/microrna/ home.do, Targetscan (http://www.targetscan.org/) and Starbase (http://starbase.sysu.edu.cn/) were employed to select the possible target circRNAs for candidate miRNAs $[26,28]$. TargetScan predicted miRNA targets based on the seed region. And miRanda was mainly based on the binding free energy of circRNAs and miRNAs, in which lower free energy indicated a stronger binding affinity between them.

\section{Binding interactions among circRNAs, miRNAs, and PGC by dual-luciferase reporter assay}

Prior to the dual-luciferase reporter assay, a transfection experiment was conducted to narrow down the range of PGC-targeting miRNAs. First, the luciferase reporter gene system was used to construct a luciferase reporter plasmid (pmitGLO-PGC) for the PGC 3'-UTR region, and the mimics of candidate miRNAs from bioinformatic analysis were also constructed (Genoarray Technology, Soochow, China). Then, they were co-transfected into HeLa cells, and the intensity of reporter gene expression 
was quantitatively measured within $48 \mathrm{~h}$. miRNAs that were positive in the above experiment were introduced into overexpression plasmids and co-transfected with the PGC 3'-UTR region reporter plasmid into AD293 cells for verification. miRNAs that were positive in both cell lines were regarded as candidate miRNAs for dual-luciferase validation experiments.

Dual-luciferase reporter assay was performed to verify the targeted binding among circRNAs, miRNAs, and PGC. Wild-type or mutant-type fragments in the PGC $3^{\prime}$-UTR associated with each candidate miRNA were designed, synthesized and inserted into GV306 vector (Genechem Co.,Ltd, Shanghai, China). Then, GV306 vectors and overexpression plasmids of candidate miRNAs were co-transfected into AD293 cells to determine the miRNA targets of the PGC gene. After $48 \mathrm{~h}$, firefly and Renilla luciferase activities were measured, and the ratio of firefly luciferase intensity to Renilla luciferase intensity was calculated (E2910; Promega). SV40-firefly_luciferase-MCS vectors carrying wild-type or mutated hsa_circ_0001483, hsa_circ_ 0001324, hsa_circ_0001051, and hsa_circ_0001614 were co-transfected with hsa-let-7c overexpression plasmid and negative control (NC) plasmid into AD293 cells to identify circRNAs with the ability to bind to hsa-let-7c (Genechem Co.,Ltd, Shanghai, China) in a targeted manner. After $48 \mathrm{~h}$, the luciferase activities of these reporters were determined using the dual-luciferase reporter assay system (E2910; Promega). The dual-luciferase reporter experiment was repeated three times in this round, and three times for each hole.

\section{Detection of circRNA, miRNA, and PGC expression in vivo and in vitro}

\section{Collection of clinical specimens and accessory information}

Gastric cancer tissue and corresponding normal tissue $(2 \mathrm{~cm}$ away from the tumor pathologically diagnosed by two pathologists) as well as accessory information were collected from 66 patients diagnosed with gastric cancer at The First Hospital of China Medical University (Shenyang; China) from November 2013 to September 2017. Enrolled subjects were pathologically diagnosed with gastric cancer by two qualified pathologists, in accordance with the World Health Organization (WHO) criteria. Patients with a history of other malignancies or undergoing preoperative radiotherapy or chemotherapy were excluded. Overall survival (OS) was followed up for 2 years. Informed consent was obtained from all participants before specimen collection. The study was approved by the ethics committee of the First Hospital of China Medical University.

\section{Cell culture}

The human GC cell lines AGS, HGC-27 and MKN-45 were purchased from the Institute of Basic Medical Sciences, Chinese Academy of Medical Sciences (Beijing; China). All cell lines were cultured in RPMI-1640 medium, with $10 \%$ fetal bovine serum (HyClone, USA). The cells were incubated at $37^{\circ} \mathrm{C}$ in a humidified atmosphere containing 5\% $\mathrm{CO}_{2}$.

\section{RNA isolation and qRT-PCR}

Total RNA was extracted using RNAiso Plus reagent, in accordance with the manufacturer's protocol (TaKaRa, Japan). cDNAs for circRNAs and mRNAs were synthesized by PrimeScript RT Master Mix with the following schedule: $37^{\circ} \mathrm{C}$ for $15 \mathrm{~min}$ and $85^{\circ} \mathrm{C}$ for $5 \mathrm{~s}$ (Perfect Real Time, cat\#RR036A; TaKaRa, Japan). Relative expression levels of circRNAs and mRNAs were determined by TB Green Premix EX Taq II (TliRNaseH Plus, cat\#RR820A; TaKaRa, Japan). For miRNAs, cDNA was synthesized by miRcute Plus miRNA First-strand cDNA Kit (cat\#KR211; TIANGEN) and the relative expression level was measured by miRcute Plus miRNA qPCR Detection Kit (cat\#FP411-02; TIANGEN). $\beta$-Actin and miR-16 were adopted as endogenous reference controls. All qRT-PCR curves were single peaks. The $2^{-\Delta \mathrm{Ct}}$ method was used to calculate the relative expression level of cDNA. The primers for circRNAs are presented in Table S1.

\section{Statistical analysis}

Statistical analysis was mainly performed by SPSSv18.0 (IBM, SPSS, and Chicago, IL, USA) and GraphPad Prism V5.0 software (GraphPad Software, USA). A $P$-value of $<0.05$ was considered to be statistically significant. Student's t-test was used to evaluate the difference between two groups for normally distributed data, while the rank sum test was applied for data with a skewed distribution. The Kaplan-Meier method was employed to estimate the association of ncRNA expression with OS, and Cox regression was performed to identify the prognostic factors of OS.

\section{Results}

\section{Identification of miRNAs targeting the PGC gene}

A total of eight bioinformatic predictive software programs were utilized to seek miRNAs targeting the PGC gene; 39 possible target miRNAs were selected (Table S2). Then, the mimics of the candidate miRNAs were co-transfected with pmirGLO-PGC plasmid into 
the tool cells and nine miRNAs were screened out upon setting a threshold of PGC down-regulation to 90\% (Table S3). The plasmids overexpressing these nine miRNAs (hsa-miR-662, hsa-miR365, hsa-let-7f, hsa-miR-98, hsa-miR-525-5p, hsa-miR-520a-5p, hsa-let-7i, hsa-miR-126-5p, and hsa-let-7c) were synthesized and co-transfected with pmirGLO-PGC. Five miRNAs (hsa-miR-365, hsa-miR-520a, hsa-let-7f, hsa-let-7c, and hsa-miR-98) were identified upon setting a threshold of PGC down-regulation to below $85 \%$ (Table S4). Dual-luciferase reporter assay showed that hsa-miR-520a, hsa-let-7c, and hsa-miR-98 could impair the luciferase activity of PGC-UTR wild-type reporter, but not the mutant type $(P=0.002, P=0.050$, and $P=0.020$, respectively; Figure 1 ).

To identify the miRNAs differentially expressed after overexpressing the PGC gene, AGS, HGC-27, and $\mathrm{MKN}-45$ cells lines were transfected individually with PGC-overexpression plasmid and NC plasmid. Compared with the level in the control group, hsa-let-7c was significantly down-regulated in the AGS cell line ( $p=0.050$, Figure $2 \mathrm{~A})$, and hsa-let-7c and hsa-miR-98 were down-regulated in the HGC-27 cell line $(P=0.011$ and $P=0.012$, respectively; Figure $2 \mathrm{~B})$, while the expression of hsa-miR-520a, hsa-let-7c, and hsa-miR-98 was not significantly changed in the MKN-45 cell line (Figure 2C). From the results of cell transfection assays, we selected hsa-let-7c for further study. Fluorescent images of the cell transfection efficiency are provided in Supplementary Figure 1 (Figure S1).

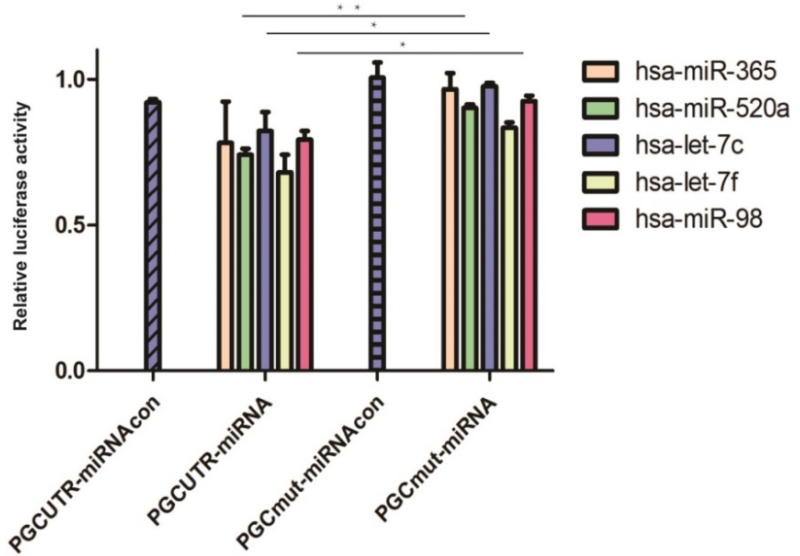

Figure 1. MiRNAs targeted to PGC in dual-luciferase reporter assay. Hsa-miR-520a, hsa-let-7c and hsa-miR-98 was significantly down-regulated in PGC wild-type group when compared with the PGC mutant-type (“***" means $p<0.010$, “*” means $p<$ $0.050)$.

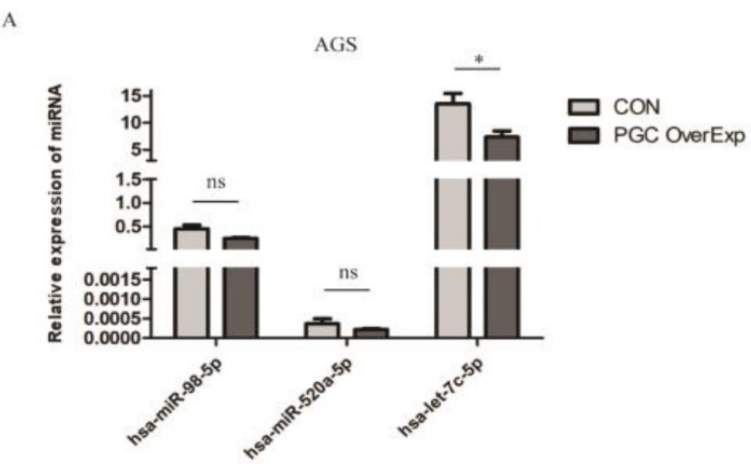

B

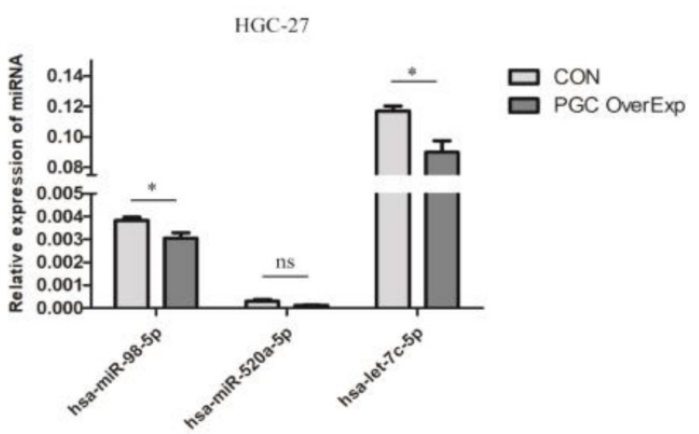

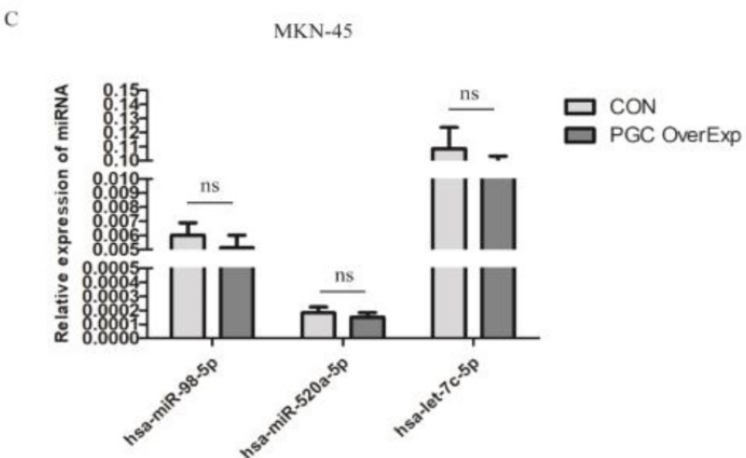

Figure 2. The expression level of hsa-let-7c when confected with PGC over-expression plasmid in AGS, HGC-27, and MKN-45 cells (“*”" means $p<0.050$; “ns” means no difference). 


\section{Identification of circRNAs targeted to PGC-related miRNAs}

Based on three bioinformatic software programs (TargetScan, miRanda, and starBase), we finally obtained 12 candidate circRNAs with targeted binding sites of hsa-let-7c for qRT-PCR validation. The expression of four of these circRNAs (hsa_circ 0012126, hsa_circ_0000365, hsa_circ_0000149, and hsa_circ_0002557) was too low in tissue to be detected by qRT-PCR, while the other seven circRNAs (hsa circ_0001483, hsa_circ_0001610, hsa_circ_0001614, hsa_circ_0001685, hsa_circ_0000504, hsa_circ_ 0001324, and hsa_circ_0001051; Table 1) showed significant differences between 30 paired GC and normal tissues. Then, four circRNAs (hsa_circ 0001614, hsa_circ_0001483, hsa_circ_0001324, and hsa_circ_0001051) with the lowest $P$-values were chosen for subsequent dual-luciferase reporter assay to validate the targeted binding relationship with hsa-let-7c. Based on the predicted binding sites from RNAhybrid, the wild type or mutant type of the four

A

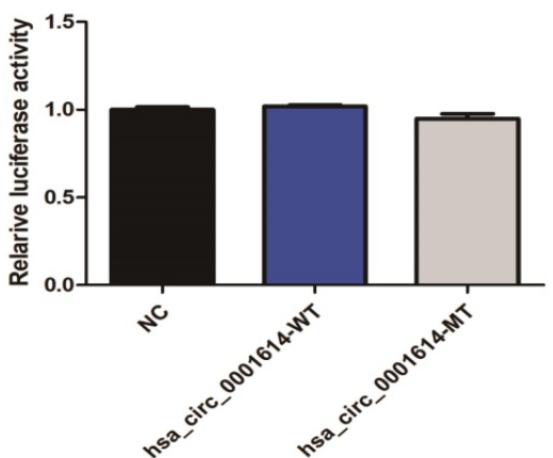

C

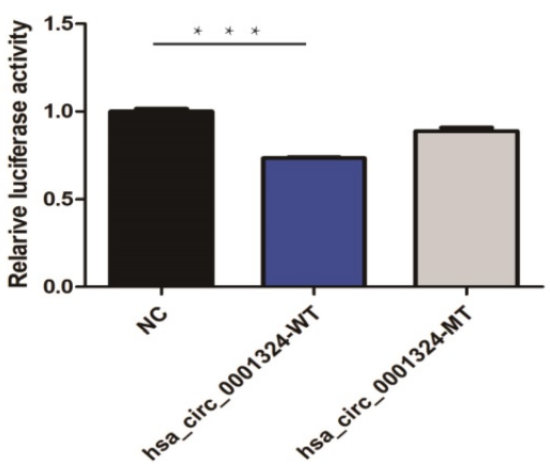

circRNA fragments was constructed, inserted downstream of the reporter gene, and co-transfected with hsa-let-7c overexpression plasmid into 293T cells. The luciferase activity was significantly reduced in hsa_circ_0001483 and hsa_circ_0001324 wild type reporter gene groups $(p=0.002$ and $p<0.001$, respectively; Figure 3).

Table 1. Expression of 8 circRNAs in 30 pairs of gastric cancer and non-cancer tissues

\begin{tabular}{|c|c|c|c|c|c|}
\hline \multirow[t]{2}{*}{ Variable } & \multicolumn{2}{|c|}{$\mathrm{CON}$} & \multicolumn{3}{|c|}{ GC } \\
\hline & $\mathrm{N}$ & $\Delta \mathrm{Ct} \mathrm{P}_{50}\left(\mathrm{P}_{25}, \mathrm{P}_{75}\right)$ & $\mathrm{N}$ & $\Delta \mathrm{Ct} \mathrm{P}_{50}\left(\mathrm{P}_{25}, \mathrm{P}_{75}\right)$ & $P$ \\
\hline hsa_circ_0001483 & 30 & $8.98(8.18,10.58)$ & 30 & $12.11(10.97,13.31)$ & 0.001 \\
\hline hsa_circ_0001610 & 30 & $5.45(4.80,6.06)$ & 30 & $6.22(5.44,7.39)$ & 0.035 \\
\hline hsa_circ_0001614 & 30 & $5.73(5.14,8.97)$ & 30 & $7.93(6.61,8.97)$ & $<0.001$ \\
\hline hsa_circ_0001685 & 30 & $7.66(7.08,8.57)$ & 30 & $9.54(8.57,10.71)$ & 0.002 \\
\hline hsa_circ_0000504 & 30 & $7.43(6.49,8.12)$ & 30 & $8.58(7.65,10.19)$ & 0.012 \\
\hline hsa_circ_0001324 & 30 & $4.78(3.76,5.86)$ & 30 & $7.37(5.97,8.52)$ & $<0.001$ \\
\hline hsa_circ_0001051 & 30 & $6.16(5.13,6.53)$ & 30 & $8.01(6.49,8.83)$ & 0.001 \\
\hline hsa_circ_0001355 & 30 & $3.63(2.64,4.28)$ & 30 & $4.51(3.11,5.73)$ & 0.250 \\
\hline
\end{tabular}

Note: This table showed the expression differences of the eight circRNAs between gastric cancer and normal tissues. The higher $\Delta \mathrm{Ct}$ value indicates the lower expression. CON, control; GC, gastric cancer.

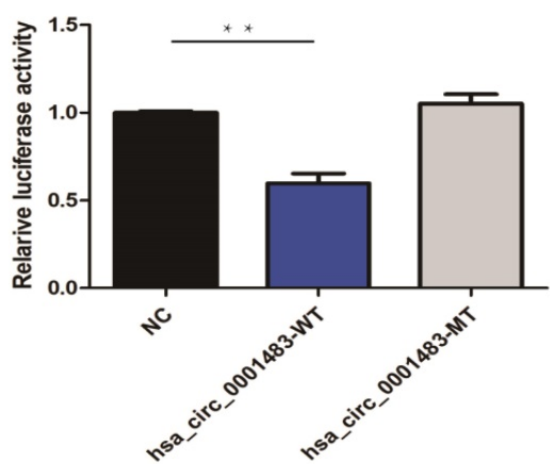

D

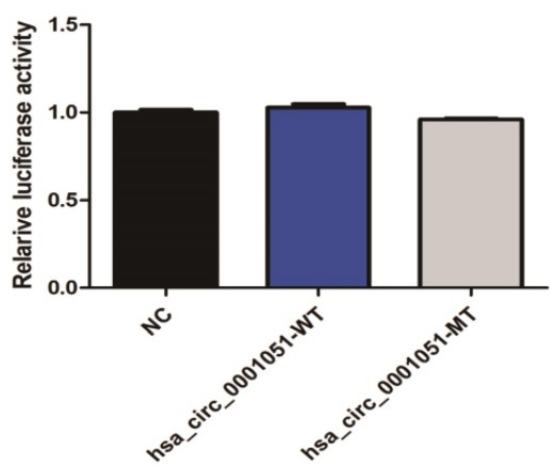

Figure 3. CircRNAs targeted to hsa-let-7c in dual-luciferase reporter assay. A, D. There was no significant reduction in luciferase reporter activity when compared hsa circ 0001614-WT type and hsa circ 0001051-WT type with control group. B, C. A significant reduction of luciferase reporter activity was observed in hsa_circ_0001483 and hsa_circ_0001324 wild-type reporter gene group than control group (“****” means $p<0.001$, “**” means $p<0.010$ ). 


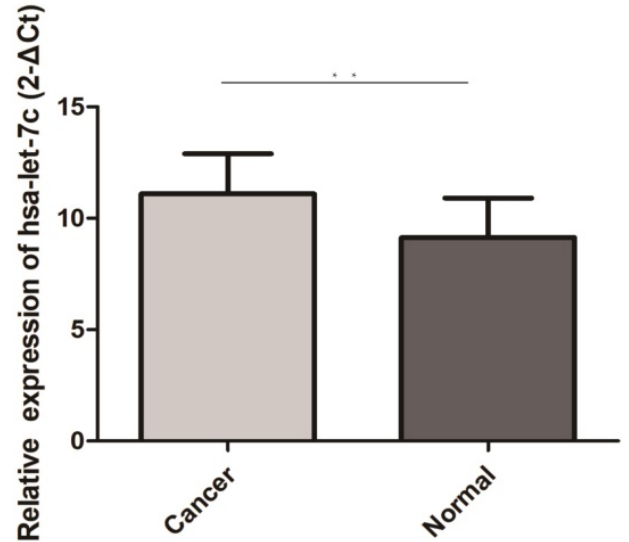

Figure 4. Hsa-let-7c was up-regulated in 30 pairs GC tissues (“***” means $p<0.010$ ).

\section{PGC-related ncRNA expression level and clinicopathological features of GC}

The qRT-PCR results of 30 paired GC and normal tissues suggested that hsa-let-7c was up-regulated in GC tissue $(p=0.003$; Figure 4$)$. The expression levels of hsa_circ_0001483, hsa_circ_ 0001324, and PGC in 66 paired GC and distant normal tissues were also determined by qRT-PCR. As shown in Figure 5, both the two circRNAs and PGC were down-regulated in GC $(p<0.001)$. Moreover, a positive correlation was found between hsa_circ_ 0001324 and PGC in GC and normal tissues $(p<0.001$,
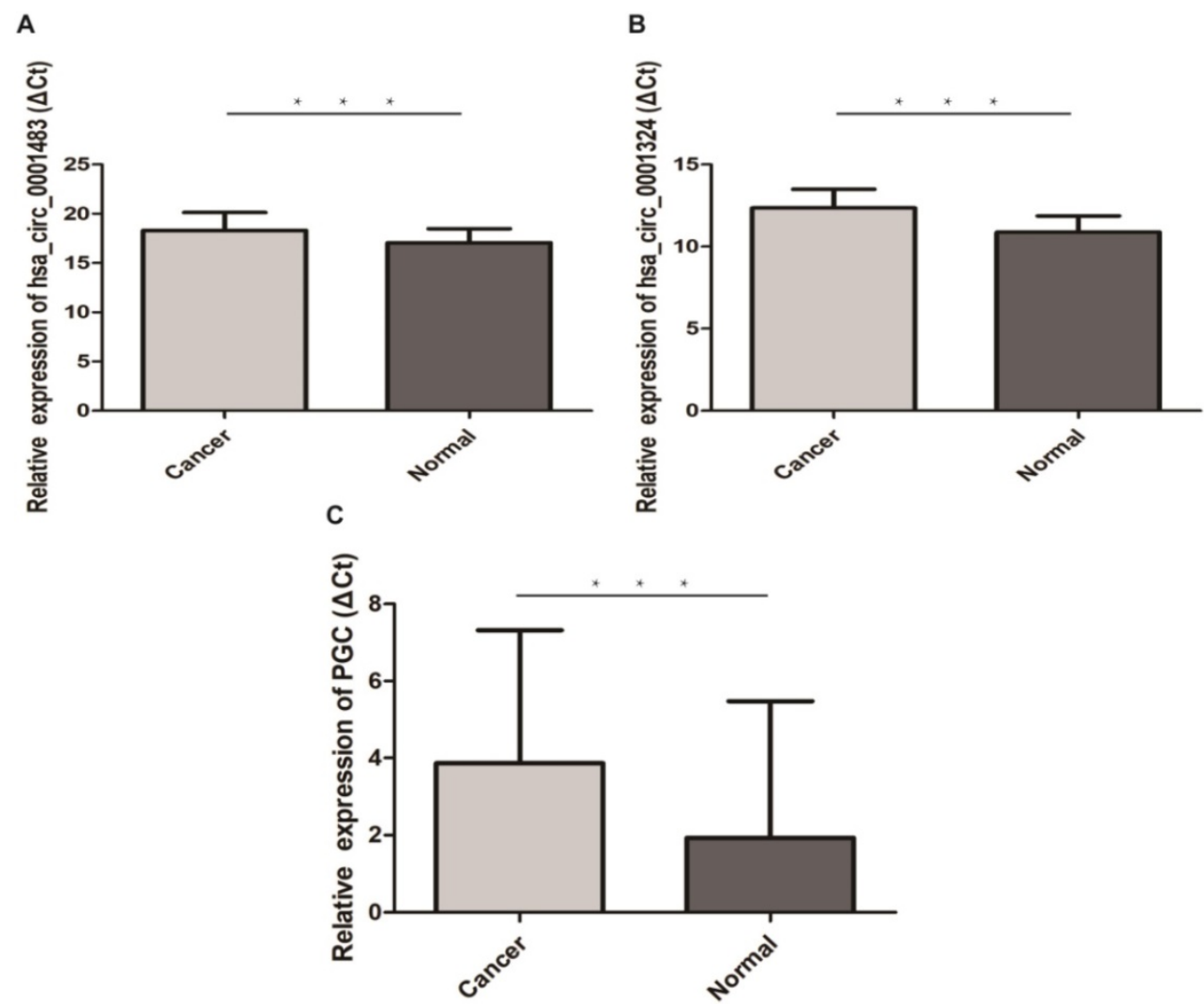

Figure 5. Hsa_circ_0001483, hsa_circ_0001324 and PGC was down-regulated in 66 pairs GC tissues ("****" means $p<0.001$ ). The higher $\Delta$ Ct value indicates a lower expression. 
Table 2. Correlation among hsa-let-7c, hsa_circ_1324, hsa_circ_ 1483 and PGC

\begin{tabular}{lllll}
\hline & \multicolumn{3}{l}{ CON } & \multicolumn{3}{l}{ GC } \\
\cline { 2 - 5 } & $\mathrm{r}$ & $P$ & $\mathrm{r}$ & $P$ \\
\hline hsa-let-7c and hsa_circ_1483 & 0.155 & 0.415 & -0.253 & 0.177 \\
hsa-let-7c and hsa_circ_1324 & 0.124 & 0.514 & 0.000 & 0.999 \\
hsa-let-7c and PGC & 0.057 & 0.766 & -0.027 & 0.888 \\
hsa_circ_1483 and PGC & $\mathbf{0 . 3 1 7}$ & $\mathbf{0 . 0 0 9}$ & 0.202 & 0.104 \\
hsa_circ_1324 and PGC & $\mathbf{0 . 4 5 6}$ & $<\mathbf{0 . 0 0 1}$ & $\mathbf{0 . 4 8 0}$ & $<\mathbf{0 . 0 0 1}$ \\
\hline
\end{tabular}

Note: The correlation analysis was performed by Spearman's correlation coefficient for the skewed distribution data. CON, control; GC, gastric cancer; PGC,

pepsinogen C; r, Spearman's correlation coefficient.

We also performed independent external validation with TCGA database. The results suggested that PGC was negatively correlated with hsa-let-7c in pathological stages I and II $(P=0.0274$, $\mathrm{r}^{2}=-0.316 ; P=0.0182, \mathrm{r}^{2}=-0.275$, respectively).

\section{Discussion}

PGC is a key gene in the process of epithelial differentiation of the stomach, and the regulatory mechanism involved in the significant downregulation of PGC expression in GC requires in-depth investigation. In the present study, miRNAs with targeted binding sites of the PGC gene and circRNAs targeting these miRNAs were predicted. Dual-luciferase reporter experiments were performed to confirm the targeted binding relationship among PGC, miRNAs, and circRNAs. The expression patterns of PGC and PGC-related ncRNAs in GC tissue were also explored by qRT-PCR. Finally, let-7c, hsa_circ_0001483, and hsa_circ_0001324 were identified as PGC-related ncRNAs in GC, and their association with the clinicopathological characteristics of GC was also revealed.

Table 3. Relationship between the expression level of hsa-let-7c, hsa_circ_0001324 and hsa_circ_0001483 in gastric cancer patients and clinicopathological factors

\begin{tabular}{|c|c|c|c|c|c|c|c|c|c|}
\hline Variability & $\begin{array}{l}\text { Patient } \\
\text { Number }\end{array}$ & $\begin{array}{l}\text { hsa_circ_0001324 }(\Delta \mathrm{Ct}) \\
\text { p50(p25, p75) }\end{array}$ & $P(2-\Delta \mathrm{Ct})$ & $\begin{array}{l}\text { Patient } \\
\text { Number }\end{array}$ & $\begin{array}{l}\text { hsa_circ_0001483( } \Delta \mathrm{Ct}) \\
\text { p50(p25, p75) }\end{array}$ & $P\left(2^{-\Delta C t}\right)$ & $\begin{array}{l}\text { Patient } \\
\text { Number }\end{array}$ & $\begin{array}{l}\text { hsa-let-7c(2- }-\Delta \mathrm{Ct}) \\
\text { p50(p25, p75) }\end{array}$ & $P(2-\Delta \mathrm{Ct})$ \\
\hline Gender & & & 0.910 & & & 0.189 & & & 0.901 \\
\hline Male & 42 & $12.36(11.41,13.77)$ & & 42 & $18.20(17.40,19.68)$ & & 16 & $3.76(0.98,10.39)$ & \\
\hline Female & 24 & $12.41(11.15,13.37)$ & & 24 & $19.11(17.78,20.61)$ & & 14 & $2.50(1.21,28.84)$ & \\
\hline Age & & & 0.005 & & & 0.586 & & & 0.917 \\
\hline$\geq 60$ & 38 & $12.65(11.81,14.10)$ & & 38 & $18.93(17.51,20.37)$ & & 13 & $2.35(1.11,14.06)$ & \\
\hline$<60$ & 28 & $11.40(10.77,12.82)$ & & 28 & $18.30(17.44,20.00)$ & & 17 & $2.97(1.29,16.53)$ & \\
\hline Smoking & & & 0.748 & & & 0.271 & & & 0.464 \\
\hline Ever Smoker & 16 & $12.30(11.67,14.19)$ & & 16 & $19.65(17.59,20.81)$ & & 4 & $1.52(0.46,36.32)$ & \\
\hline Never Smoker & 50 & $12.43(11.26,13.26)$ & & 50 & $18.27(17.44,19.70)$ & & 26 & $2.97(1.21,12.27)$ & \\
\hline Drinking & & & 0.758 & & & 0.433 & & & 0.454 \\
\hline Ever Drinker & 9 & $11.72(11.68,14.28)$ & & 9 & $19.67(17.07,20.93)$ & & 2 & NA & \\
\hline Never Drinker & 57 & $12.44(11.26,13.39)$ & & 57 & $18.27(17.50,19.92)$ & & 28 & $2.97(1.16,15.84)$ & \\
\hline Family History & & & 0.866 & & & 0.449 & & & 0.707 \\
\hline Yes & 15 & $12.52(11.22,14.05)$ & & 15 & $18.59(18.24,20.47)$ & & 8 & $2.33(1.46,4.62)$ & \\
\hline No & 51 & $12.22(11.28,13.34)$ & & 51 & $18.15(17.44,20.07)$ & & 22 & $3.31(0.96,26.33)$ & \\
\hline Location & & & 0.767 & & & 0.418 & & & 0.891 \\
\hline Body & 20 & $12.28(11.29,13.41)$ & & 20 & $18.09(16.94,19.66)$ & & 13 & $2.03(1.28,7.62)$ & \\
\hline Angle & 11 & $11.84(11.11,12.64)$ & & 11 & $19.30(18.47,21.65)$ & & 3 & NA & \\
\hline Antrum & 23 & $12.60(11.24,13.84)$ & & 23 & $18.26(17.78,19.67)$ & & 10 & $2.66(1.40,44.06)$ & \\
\hline Entire & 11 & $12.03(11.31,14.05)$ & & 11 & $19.59(17.00,20.61)$ & & 3 & NA & \\
\hline Macroscopic Type & & & 0.856 & & & 0.718 & & & 0.565 \\
\hline Protruded Type & 3 & NA & & 3 & NA & & 2 & NA & \\
\hline Ulcerative Type & 13 & $12.44(11.75,12.86)$ & & 13 & $18.60(17.17,20.06)$ & & 6 & $2.15(0.88,16.99)$ & \\
\hline Ulcerative Infiltrative Type & 32 & $12.37(10.72,13.30)$ & & 32 & $18.53(17.88,20.22)$ & & 13 & $5.17(1.57,16.53)$ & \\
\hline Diffuse Infiltrative Type & 18 & $11.87(11.27,14.15)$ & & 18 & $18.30(16.77,19.77)$ & & 9 & $2.97(1.18,32.08)$ & \\
\hline Histological Type & & & 0.744 & & & 0.287 & & & 0.499 \\
\hline Papillary Adenocarcinoma (I) & 0 & NA & & 0 & NA & & 0 & NA & \\
\hline Well Differentiated Type (II) & 1 & NA & & 1 & NA & & 1 & NA & \\
\hline Moderately Differentiated Type (III) & 8 & $12.39(11.84,12.90)$ & & 8 & $18.07(17.53,20.10)$ & & 2 & NA & \\
\hline Poorly Differentiated Type (IV) & 39 & $12.38(11.24,14.09)$ & & 32 & $18.33(17.42,19.77)$ & & 15 & $1.69(1.23,8.51)$ & \\
\hline Mucinous Adenocarcinoma (V) & 6 & $12.73(10.91,13.30)$ & & 5 & $20.04(18.57,20.52)$ & & 4 & $2.50(0.92,8.34)$ & \\
\hline Signet-ring Cell Cinoma (VI) & 10 & $11.43(10.88,13.07)$ & & 9 & $17.87(16.85,19.18)$ & & 6 & $26.63(3.37,76.49)$ & \\
\hline Lauren Classification & & & 0.757 & & & 0.915 & & & 0.577 \\
\hline Intestinal & 9 & $12.33(11.84,12.77)$ & & 9 & $18.15(17.62,19.84)$ & & 3 & NA & \\
\hline Diffuse & 55 & $12.22(11.22,13.84)$ & & 55 & $18.47(17.42,20.07)$ & & 25 & $2.97(1.28,13.88)$ & \\
\hline TNM stage & & & 0.473 & & & 0.160 & & & 0.422 \\
\hline I & 7 & $12.01(10.71,12.64)$ & & 7 & $19.30(18.26,22.69)$ & & 4 & $4.11(0.78,9.54)$ & \\
\hline II & 23 & $12.86(11.11,14.35)$ & & 23 & $18.15(17.34,20.37)$ & & 9 & $10.13(1.34,55.27)$ & \\
\hline III & 36 & $12.22(11.34,13.30)$ & & 36 & $18.30(17.44,19.70)$ & & 17 & $2.03(1.18,6.84)$ & \\
\hline \multirow[t]{2}{*}{ IV } & 0 & NA & & 0 & NA & & 0 & NA & \\
\hline & & & 0.606 & & & 0.575 & & & 0.315 \\
\hline $\mathrm{I}+\mathrm{II}$ & 30 & $12.54(11.07,13.95)$ & & 30 & $18.54(17.51,21.06)$ & & 13 & $6.73(1.24,29.72)$ & \\
\hline $\mathrm{III}+\mathrm{IV}$ & 36 & $12.22(11.34,13.30)$ & & 36 & $18.30(17.44,19.70)$ & & 17 & $2.03(1.18,6.84)$ & \\
\hline Peritumoral Inflammatory Cells & & & 0.685 & & & 0.03 & & & 0.159 \\
\hline
\end{tabular}




\begin{tabular}{|c|c|c|c|c|c|c|c|c|c|}
\hline Variability & $\begin{array}{l}\text { Patient } \\
\text { Number }\end{array}$ & $\begin{array}{l}\text { hsa_circ_0001324 }(\Delta \mathrm{Ct}) \\
\text { p50(p25, p75) }\end{array}$ & $P\left(2^{-\Delta C t}\right)$ & $\begin{array}{l}\text { Patient } \\
\text { Number }\end{array}$ & $\begin{array}{l}\text { hsa_circ_0001483( } \Delta \mathrm{Ct}) \\
\text { p50(p25, p75) }\end{array}$ & $P\left(2^{-\Delta C t}\right)$ & $\begin{array}{l}\text { Patient } \\
\text { Number }\end{array}$ & $\begin{array}{l}\text { hsa-let-7c(2- }-\Delta \mathrm{Ct}) \\
\text { p50(p25, p75) }\end{array}$ & $P(2-\Delta \mathrm{Ct})$ \\
\hline+ & 18 & $11.97(11.29,12.89)$ & & 18 & $17.80(16.85,18.68)$ & & 9 & $8.51(2.66,42.26)$ & \\
\hline++ & 26 & $12.41(11.02,13.42)$ & & 26 & $19.30(18.15,20.62)$ & 0.02 & 11 & $1.69(0.99,5.17)$ & \\
\hline+++ & 21 & $12.60(11.42,14.15)$ & & 21 & $18.32(17.40,20.43)$ & & 9 & $1.44(1.00,10.30)$ & \\
\hline Lymphovascular invasion & & & 0.931 & & & 0.984 & & & 0.485 \\
\hline+ & 42 & $12.37(11.26,13.77)$ & & 42 & $18.33(17.43,20.15)$ & & 18 & $2.19(1.10,8.91)$ & \\
\hline- & 24 & $12.36(11.35,13.29)$ & & 24 & $18.43(17.48,20.20)$ & & 12 & $4.85(1.36,33.11)$ & \\
\hline Ganglion invasion & & & 0.352 & & & 0.375 & & & 0.518 \\
\hline+ & 53 & $12.09(11.23,13.74)$ & & 53 & $18.32(17.37,20.38)$ & & 23 & $2.97(1.34,22.94)$ & \\
\hline- & 12 & $12.58(12.09,13.51)$ & & 12 & $18.53(18.06,19.59)$ & & 6 & $4.11(0.79,10.22)$ & \\
\hline Lymphatic metastasis & & & 0.332 & & & 0.044 & & & 0.944 \\
\hline+ & 45 & $12.22(11.25,13.39)$ & & 45 & $18.27(17.42,19.67)$ & & 22 & $2.19(1.21,12.00)$ & \\
\hline- & 21 & $12.64(11.66,13.76)$ & & 21 & $19.30(17.87,22.14)$ & & 8 & $4.85(0.78,19.83)$ & \\
\hline Depth of invasion & & & 0.583 & & & 0.264 & & & 0.937 \\
\hline Mucous Layer (pT1) & 2 & NA & & 2 & NA & & 1 & NA & \\
\hline Submucosal Layer (pT2) & 5 & $11.48(10.34,12.68)$ & & 5 & $18.03(17.06,18.10)$ & & 3 & NA & \\
\hline Muscular Layer (pT3) & 7 & $12.52(11.11,12.86)$ & & 7 & $18.88(18.47,22.69)$ & & 3 & NA & \\
\hline Subserosa Layer (pT4) & 1 & NA & & 1 & NA & & 0 & NA & \\
\hline \multirow{2}{*}{$\begin{array}{l}\text { Serosal Layer or Invasion Adjacent } \\
\text { Organs(pT5) }\end{array}$} & 51 & $12.52(11.28,14.09)$ & & 43 & $18.32(17.40,20.37)$ & & 23 & $2.35(1.23,22.94)$ & \\
\hline & & & 0.340 & & & 0.263 & & & 0.845 \\
\hline $\mathrm{pT} 1+\mathrm{pT} 2$ & 7 & $1201(10.37,12.44)$ & & 7 & $18.04(17.45,18.26)$ & & 4 & $2.23(1.02,5.79)$ & \\
\hline $\mathrm{pT} 3+\mathrm{pT} 4$ & 8 & $12.18(11.19,12.81)$ & & 8 & $19.09(18.50,21.86)$ & & 3 & NA & \\
\hline pT5 & 51 & $12.52(11.28,14.09)$ & & 51 & $18.32(17.40,20.37)$ & & 23 & $2.34(1.23,22.94)$ & \\
\hline Growth Pattern & & & 0.904 & & & 0.938 & & & 0.067 \\
\hline Massive or Nested & 11 & $12.52(10.31,13.34)$ & & 11 & $18.15(17.78,20.38)$ & & 26 & $3.63(1.31,18.96)$ & \\
\hline Diffuse Infiltrative or Infiltralive & 55 & $12.22(11.28,13.67)$ & & 55 & $18.47(17.42,20.07)$ & & 4 & $1.17(0.45,1.89)$ & \\
\hline
\end{tabular}

Note: $\mathrm{CON}$, control; GC, gastric cancer. The higher $\Delta \mathrm{Ct}$ value indicates a lower expression while the higher $2^{-\Delta \mathrm{Ct}}$ value means the higher expression level.

Dysregulation of ncRNAs has been recognized as a novel molecular signature in cancer initiation and development. As a type of ncRNAs, miRNAs play an important role in down-regulating the transcription of target mRNAs. The relationship of miRNAs with GC has been demonstrated in previous reports [29]. For example, miR-92a-1-5p was shown to increase CDX2 by targeting FOXD1 and to mediate gastric intestinal metaplasia [30]. miR-143-3p may inhibit GC growth and confer greater sensitivity sensitive to cisplatin by targeting BRD2 [31]. Our study focused on the ncRNAs down-regulating PGC in GC tissue. Dual-luciferase reporter assay confirmed the findings of three miRNAs predicted by software to bind to PGC (hsa-miR-520a, hsa-let-7c, and hsa-miR-98). Accordingly, hsa-miR-520 was indicated to regulate the proliferation and migration of lung cancer and also modulate the progression of renal carcinoma. However, there was a lack of evidence that this miRNA is related to GC $[32,33]$. miR-98 could serve as a biomarker for prostate cancer diagnosis. It might be up-regulated in GC and thus involved in GC progression $[34,35]$. The Let-7 miRNA family plays a central role in regulating the relationship between miRNAs and mRNAs in GC, and hsa-let-7c was shown to be associated with the clinicopathological parameters of GC [36]. Here, the above three miRNAs were first reported to be involved in PGC tissue expression. Our preliminary research showed that the serum hsa-let-7c expression level was inversely related to PGC [37]. These PGC-related miRNAs may be involved in GC development by regulating PGC expression, although the specific mechanisms involved remain to be clarified. Nonetheless, they are potential biomarkers for GC.

circRNAs are another type of ncRNAs with unclear roles in cancer. They have been suggested to function as miRNA sponges in various cancers and are potential novel biomarkers [38-40]. Many studies have reported that circRNAs exert regulatory roles in GC. Rong et al found that circHECTD1 activated $\beta$-catenin/c-Myc signaling by the adsorption of miR-1256, facilitated glutaminolysis, and thus promoted GC progression [41]. In addition, circAKT3 was found to enhance cisplatin resistance in GC by suppressing hsa-miR-198 to up-regulate PIK3R1 [42]. In this study, the target circRNAs for hsa-let-7c were predicted by bioinformatic methods and 12 candidates were validated by qRT-PCR in 30 pairs of GC tissues. We selected four down-regulated circRNAs (hsa_circ_0001483, hsa_circ_0001324, hsa_circ-0001614, and hsa_circ_0001051) $(p<0.001)$ for dual-luciferase reporter assay with hsa-let-7c. The results showed that hsa_circ_0001483 and hsa circ_0001324 could combine with hsa-let-7c. Based on dual-luciferase reporter assay, the PGC-specific ceRNA network was constructed, including hsa_circ 0001483/hsa_circ_0001324, hsa-let-7c, and PGC in GC. Consequently, hsa_circ_0001483 and hsa_circ_ 0001324 might down-regulate PGC expression by competitively binding to hsa-let-7c, and the two novel circRNAs could also serve as biomarkers for GC diagnosis. 
The expression levels of hsa-let-7c, hsa_circ 0001483, hsa_circ_0001324, and PGC in GC tissues and distant normal tissues were further investigated by qRT-PCR. It was found that hsa_circ_0001483 and hsa_circ_0001324 were significantly down-regulated in GC tissue and had collinearity with PGC expression, indicating that their low expression in GC was consistent with the low expression of PGC. Moreover, hsa-let-7c was significantly overexpressed in GC tissues. These findings suggested possible endogenous interactions among these ncRNAs and the PGC gene. The abnormally low expression of hsa_circ_0001483 and hsa_circ_0001324 with high expression of hsa-let-7c in GC tissue makes more hsa-let-7c binds to the PGC-UTR region, leading to the down regulated expression of PGC. Furthermore, the expression level of hsa_circ_0001483 was associated with pericarcinoma inflammatory cell infiltration and lymphatic metastasis. Immune cell infiltration and metastasis are key events worthy of consideration when evaluating GC prognosis and therapeutic strategy [43-45]. The dysregulation of hsa_circ_0001483 may influence immune response, chemosensitivity-related features, and the metastasis of GC patients, making it a potential biomarker for GC prognosis. In addition, higher expression of hsa circ_0001324 and hsa_circ_0001483 was suggestive of better OS $(p=0.070$ and $p=0.081$, respectively). Considering a previous report describing that a low PGC expression level was correlated with shorter OS of GC patients [46], we believed that the low expression of hsa_circ_0001483 and hsa_circ_0001324 attenuated their capacity to absorb hsa-let-7c, thus down-regulating PGC expression, which may affect the prognosis of GC patients.

Some limitations of this study should be acknowledged. First, no complete analysis of the upand down-stream targets involved in the related mechanisms was performed in this study, which could be focus of future research. In addition, our findings also require more independent validation by an external cohort, which warrant future investigation.

In conclusion, the PGC-related ncRNAs and their association with the clinicopathological parameters of GC were reported here for the first time. hsa_circ_0001483, hsa_circ_0001324, and let-7c were newly identified and validated as PGC-related ncRNAs, and they showed various associations with the clinicopathological features of GC. The hsa_circ 0001483/hsa_circ_0001324-hsa-let-7c-PGC axis might account for PGC down-regulation in GC tissue. Identification of the underlying molecular mechanisms underlying GC is of great significance for detecting therapeutic targets for management strategies. More importantly, further exploration of the biological functions of PGC, hsa-let-7c, as well as hsa_circ_0001324 and hsa_circ_0001483 could deepen our understanding of the pathogenesis of GC and also improve the diagnosis and treatment of this disease.

\section{Supplementary Material}

Supplementary figure and tables. http://www.jcancer.org/v12p4389s1.pdf

\section{Acknowledgements}

The authors gratefully acknowledge financial support from the National Nature Science Foundation of China (Ref No. 81772987) and Natural Science Foundation of Liaoning Province in China (Ref No. 20170540987).

\section{Funding}

This work is supported partly by grants from the National Nature Science Foundation of China (Ref No. 81772987) and Natural Science Foundation of Liaoning Province in China (Ref No. 20170540987).

\section{Author Contributions}

$Y Y$ and $X Q$ provide direction and guidance throughout the preparation of this manuscript. XQ and $\mathrm{DH}$ completed experiments and data statistics. $W Y$ repeated the luciferase experiments independently. DH drafted the manuscript. YY reviewed the manuscript and made significance revisions on the drafts. All authors read and approved the final manuscript.

\section{Abbreviations}

PGC: Pepsinogen C; GC: gastric cancer; PG: pepsinogen; SG: superficial gastritis; AG: atrophic gastritis; 3'-UTR: 3'-Untranslated region; MRE: MiRNA response element; NC: Negative control; OS: Overall survival.

\section{Competing Interests}

The authors have declared that no competing interest exists.

\section{References}

1. Shen S, Jiang J, Yuan Y. Pepsinogen C expression, regulation and its relationship with cancer. Cancer Cell Int. 2017; 17: 57.

2. Ning PF, Liu HJ, Yuan Y. Dynamic expression of pepsinogen C in gastric cancer, precancerous lesions and Helicobacter pylori associated gastric diseases. World J Gastroenterol. 2005; 11: 2545-8.

3. Ishihara T, Ichihara Y, Hayano T, Katsura I, Sogawa K, Fujii-Kuriyama Y, et al. Primary structure and transcriptional regulation of rat pepsinogen $\mathrm{C}$ gene. J Biol Chem. 1989; 264: 10193-9.

4. Dong Y, Kang H, Liu H, Wang J, Guo Q, Song C, et al. Myoferlin, a Membrane Protein with Emerging Oncogenic Roles. Biomed Res Int. 2019; 2019: 7365913.

5. Massarrat S, Haj-Sheykholeslami A, Mohamadkhani A, Zendehdel N, Aliasgari A, Rakhshani N, et al. Pepsinogen II can be a potential surrogate marker of morphological changes in corpus before and after $\mathrm{H}$. pylori eradication. Biomed Res Int. 2014; 2014: 481607. 
6. Jiang J, Shen S, Dong N, Liu J, Xu Q, Sun L, et al. Correlation between negative expression of pepsinogen $C$ and a series of phenotypic markers of gastric cancer in different gastric diseases. Cancer Med. 2018; 7: 4068-76.

7. Melle C, Ernst G, Schimmel B, Bleul A, Kaufmann R, Hommann M, et al. Characterization of pepsinogen $C$ as a potential biomarker for gastric cancer using a histo-proteomic approach. J Proteome Res. 2005; 4: 1799-804.

8. Jin G, Ma H, Wu C, Dai J, Zhang R, Shi Y, et al. Genetic variants at 6 p21.1 and 7 p15.3 are associated with risk of multiple cancers in Han Chinese. Am J Hum Genet. 2012; 91: 928-34.

9. Liu Y, Guo R, Hao G, Xiao J, Bao Y, Zhou J, et al. The expression profiling and ontology analysis of noncoding RNAs in peritoneal fibrosis induced by peritoneal dialysis fluid. Gene. 2015; 564: 210-9.

10. Li C, Wang Z, Zhang J, Zhao X, Xu P, Liu X, et al. Crosstalk of mRNA, miRNA, IncRNA, and circRNA and Their Regulatory Pattern in Pulmonary Fibrosis. Mol Ther Nucleic Acids. 2019; 18: 204-18.

11. Gu Y, Zhao S, Wan J, Meng J, Zuo C, Wang S, et al. Hsa-miRNA-125b may induce apoptosis of HTR8/SVneo cells by targeting MCL1. Reprod Biol. 2019; 19: 368-73.

12. Lin S, Gregory RI. MicroRNA biogenesis pathways in cancer. Nat Rev Cancer. 2015; 15: 321-33.

13. Liu L, Wang J, Khanabdali R, Kalionis B, Tai X, Xia S. Circular RNAs: Isolation, characterization and their potential role in diseases. RNA Biol. 2017; 14: 1715-21.

14. Zhang Y, Han T, Li J, Cai H, Xu J, Chen L, et al. Comprehensive analysis of the regulatory network of differentially expressed mRNAs, lncRNAs and circRNAs in gastric cancer. Biomed Pharmacother. 2020; 122: 109686.

15. Sibbald IR, Wolynetz MS. True and apparent metabolisable energy. Br Poult Sci. 1987; 28: 782-8.

16. Liu WJ, Lv Z, Liu JW, Xu Q, Ding HX, Yuan Y. LncRNAs adjacent to pepsinogen $C$ in gastric diseases and diagnostic efficiencies of joint detection in gastric diseases. Future Oncol. 2020; 16: 655-63.

17. Lv Z, Sun L, Xu Q, Gong Y, Jing J, Dong N, et al. SNP interactions of PGC with its neighbor lncRNAs enhance the susceptibility to gastric cancer/atrophic gastritis and influence the expression of involved molecules. Cancer Med. 2018; 7: 5252-71

18. Xu Q, Wu YF, Li Y, He CY, Sun LP, Liu JW, et al. SNP-SNP interactions of three new pri-miRNAs with the target gene PGC and multidimensional analysis of $\mathrm{H}$. pylori in the gastric cancer/atrophic gastritis risk in a Chinese population. Oncotarget. 2016; 7: 23700-14.

19. Wu YF, Xu Q, He CY, Li Y, Liu JW, Deng N, et al. Association of Polymorphisms in three pri-miRNAs that Target Pepsinogen $\mathrm{C}$ with the Risk and Prognosis of Gastric Cancer. Sci Rep. 2017; 7: 39528

20. Hsu SD, Lin FM, Wu WY, Liang C, Huang WC, Chan WL, et al. miRTarBase: a database curates experimentally validated microRNA-target interactions. Nucleic Acids Res. 2011; 39: D163-9.

21. Maragkakis M, Vergoulis T, Alexiou P, Reczko M, Plomaritou K, Gousis M, et al. DIANA-microT Web server upgrade supports Fly and Worm miRNA target prediction and bibliographic miRNA to disease association. Nucleic Acids Res. 2011; 39: W145-8.

22. Betel D, Wilson M, Gabow A, Marks DS, Sander C. The microRNA.org resource: targets and expression. Nucleic Acids Res. 2008; 36: D149-53.

23. Wong N, Wang X. miRDB: an online resource for microRNA target prediction and functional annotations. Nucleic Acids Res. 2015; 43: D146-52.

24. Bordley JE. The American Rhinologic Society: its foundation and its future responsibilities. Address of the guest of honor. Laryngoscope. 1980; 90: 876-9.

25. Rela M, Heaton ND. Split-liver transplantation. Br J Surg. 1998; 85: 881-3.

26. Chiang HR, Schoenfeld LW, Ruby JG, Auyeung VC, Spies N, Baek D, et al. Mammalian microRNAs: experimental evaluation of novel and previously annotated genes. Genes Dev. 2010; 24: 992-1009.

27. Yong MS, Marks GS. Studies of the chemical nature of the alpha-adrenergic receptor. IV. Labeling studies on nerve-free rabbit aortic strips. Biochem Pharmacol. 1969; 18: 1619-26.

28. John B, Enright AJ, Aravin A, Tuschl T, Sander C, Marks DS. Human MicroRNA targets. PLoS Biol. 2004; 2: e363.

29. Cho WC. OncomiRs: the discovery and progress of microRNAs in cancers. Mol Cancer. 2007; 6: 60.

30. Li T, Guo H, Li H, Jiang Y, Zhuang K, Lei C, et al. MicroRNA-92a-1-5p increases CDX2 by targeting FOXD1 in bile acids-induced gastric intestinal metaplasia. Gut. 2019; 68: 1751-63.

31. Chen Z, Li Z, Soutto M, Wang W, Piazuelo MB, Zhu S, et al. Integrated Analysis of Mouse and Human Gastric Neoplasms Identifies Conserved microRNA Networks in Gastric Carcinogenesis. Gastroenterology. 2019; 156: 1127-39 e8.

32. Moradi Marjaneh R, Khazaei M, Ferns GA, Avan A, Aghaee-Bakhtiari SH. MicroRNAs as potential therapeutic targets to predict responses to oxaliplatin in colorectal cancer: From basic evidence to therapeutic implication. IUBMB Life. 2019; 71: 1428-41.

33. Zhou W, Wu Y, Pan M, Liu D, Liu B. Proliferation and Migration of Lung Cancer Could be Inhibited by Oxymatrine through the Regulation for miR-520/VEGF. Am J Chin Med. 2019; 47: 865-78.

34. Moya L, Meijer J, Schubert S, Matin F, Batra J. Assessment of miR-98-5p, miR-152-3p, miR-326 and miR-4289 Expression as Biomarker for Prostate Cancer Diagnosis. Int J Mol Sci. 2019; 20.

35. Yao Y, Suo AL, Li ZF, Liu LY, Tian T, Ni L, et al. MicroRNA profiling of human gastric cancer. Mol Med Rep. 2009; 2: 963-70.
36. Yepes S, Lopez R, Andrade RE, Rodriguez-Urrego PA, Lopez-Kleine L, Torres MM. Co-expressed miRNAs in gastric adenocarcinoma. Genomics. 2016; 108: 93-101.

37. Liu WJ, Xu Q, Sun LP, Dong QG, He CY, Yuan Y. Expression of serum let-7c, let-7i, and let-7f microRNA with its target gene, pepsinogen $C$, in gastric cancer and precancerous disease. Tumour Biol. 2015; 36: 3337-43.

38. Hansen TB, Jensen TI, Clausen BH, Bramsen JB, Finsen B, Damgaard CK, et al. Natural RNA circles function as efficient microRNA sponges. Nature. 2013; 495: 384-8.

39. Ashwal-Fluss R, Meyer M, Pamudurti NR, Ivanov A, Bartok O, Hanan M, et al. circRNA biogenesis competes with pre-mRNA splicing. Mol Cell. 2014; 56: 55-66.

40. Su H, Lin F, Deng X, Shen L, Fang Y, Fei Z, et al. Profiling and bioinformatics analyses reveal differential circular RNA expression in radioresistant esophageal cancer cells. J Transl Med. 2016; 14: 225.

41. Cai J, Chen Z, Wang J, Wang J, Chen X, Liang L, et al. circHECTD1 facilitates glutaminolysis to promote gastric cancer progression by targeting miR-1256 and activating beta-catenin/c-Myc signaling. Cell Death Dis. 2019; 10: 576.

42. Huang X, Li Z, Zhang Q, Wang W, Li B, Wang L, et al. Circular RNA AKT3 upregulates PIK3R1 to enhance cisplatin resistance in gastric cancer via miR-198 suppression. Mol Cancer. 2019; 18: 71.

43. Li L, Ouyang Y, Wang W, Hou D, Zhu Y. The landscape and prognostic value of tumor-infiltrating immune cells in gastric cancer. PeerJ. 2019; 7: e7993.

44. Jiang $\mathrm{Y}$, Xie J, Huang $\mathrm{W}$, Chen $\mathrm{H}$, Xi S, Han $\mathrm{Z}$, et al. Tumor Immune Microenvironment and Chemosensitivity Signature for Predicting Response to Chemotherapy in Gastric Cancer. Cancer Immunol Res. 2019; 7: 2065-73.

45. Yang $\mathrm{Y}$, Shao $\mathrm{Y}, \mathrm{Zhu} \mathrm{M}, \mathrm{Li}$ Q, Yang F, Lu X, et al. Using gastric juice lncRNA-ABHD11-AS1 as a novel type of biomarker in the screening of gastric cancer. Tumour Biol. 2016; 37: 1183-8.

46. Fernandez R, Vizoso F, Rodriguez JC, Merino AM, Gonzalez LO, Quintela I, et al. Expression and prognostic significance of pepsinogen $\mathrm{C}$ in gastric carcinoma. Ann Surg Oncol. 2000; 7: 508-14. 